Revue de droit comparé du travail et de la sécurité sociale

$3 \mid 2020$

La Directive 2019/1158 du 20 juin 2019 concernant l'équilibre entre vie personnelle et vie privée des parents et des aidants

\title{
Interprétation de la récente réglementation de la grève en Uruguay dans une perspective constitutionnelle et internationale
}

\section{Hugo Barretto Ghione}

\section{OpenEdition \\ Journals}

Édition électronique

URL : https://journals.openedition.org/rdctss/1030

DOI : $10.4000 /$ rdctss. 1030

ISSN : 2262-9815

\section{Éditeur}

Centre de droit comparé du travail et de la sécurité sociale

\section{Édition imprimée}

Date de publication : 1 novembre 2020

Pagination : 184-187

ISSN : $2117-4350$

\section{Référence électronique}

Hugo Barretto Ghione, «Interprétation de la récente réglementation de la grève en Uruguay dans une perspective constitutionnelle et internationale », Revue de droit comparé du travail et de la sécurité sociale [En ligne], 3 | 2020, mis en ligne le 01 novembre 2021, consulté le 11 novembre 2021. URL: http://journals.openedition.org/rdctss/1030 ; DOI : https://doi.org/10.4000/rdctss.1030

Revue de droit comparé du travail et de la sécurité sociale est mise à disposition selon les termes de la Licence Creative Commons Attribution - Pas d'Utilisation Commerciale - Pas de Modification 4.0 International. 


\section{HUGO BARRETTO GHIONE}

FACULTÉ DE DROIT DE L'UNIVERSITÉ DE LA RÉPUBLIQUE

\section{INTERPRÉTATION DE LA RÉCENTE RÉGLEMENTATION DE LA GRÈVE EN URUGUAY DANS UNE PERSPECTIVE CONSTITUTIONNELLE ET INTERNATIONALE}

\section{L'« URGENCE » DE RÉGLEMENTER LA GRÈVE}

II suffit d'étudier la récente réglementation (partielle) du droit de grève en Uruguay pour se rendre compte des contraintes que les changements politiques font peser sur le droit social, et en particulier sur le droit du travail.

Le gouvernement qui est entré en fonction le 1er mars 2020 - composé d'une coalition de partis traditionnels de centre droit - a promu une loi $\mathrm{d}^{\prime}$ " urgente considération » dont 476 dispositions réglementent des questions aussi diverses que : la sécurité publique - prévoyant des sanctions pénales plus lourdes -, l'éducation, la fiscalité, les entreprises publiques, l'efficacité de l'État et les achats publics, le statut des fonctionnaires, la politique du logement, les soins de santé à prix élevé, la réforme de la sécurité sociale, les règles relatives aux baux immobiliers, certaines modifications du Code civil et la grève pour ne citer que quelques exemples.

Le mécanisme constitutionnel de la « Déclaration d'urgente considération » (art. 168, al. 7) donne à l'exécutif le pouvoir de soumettre un projet de loi au Parlement national pour examen dans de très brefs délais, avec pour lourde conséquence qu'il sera réputé approuvé « s'il n'a pas été expressément rejeté dans ces délais [Ndlr : environ 90 jours] et qu'un projet de loi de remplacement n'a pas été approuvé ". Le fait que le pouvoir exécutif ait soumis un projet au contenu si varié pour un examen dans un délai si court, et en plus au moment le plus critique de la pandémie de COVID 19, a suscité de vives critiques de la part des universitaires ${ }^{1}$ comme de l'opposition politique et sociale puisque, comme on peut le voir dans la liste récapitulative dressée ci-dessus, le projet envisageait dans de nombreux cas la restriction des droits fondamentaux des individus, ce qui ne peut être défini de manière aussi légère.

Les groupes qui ont émis des critiques ont par exemple constaté que l'adoption de la plupart des dispositions envisagées dans le projet ne revêtait pas un tel caractère "d'urgence », si l'on entend par ce terme la nécessité impérieuse et pressante de résoudre une question au plus vite, conformément à la définition que l'on trouve dans n'importe quel dictionnaire de la langue espagnole.

La seule urgence résidait dans la volonté politique du gouvernement d'approuver un texte qui guiderait ses actions dans les années à venir. Si le projet a fait l'objet de critiques

1 L'Université de la République, établissement d'enseignement supérieur le plus important du pays, a été très critique à l'égard des solutions proposées dans le cadre du projet ainsi que du moment choisi pour le présenter, considéré comme opportuniste. 
fondées - sa constitutionnalité ayant même été remise en cause - cela n'a pas pour autant empêché la majorité parlementaire d'adopter ce texte « d'urgente considération » en tant que loi nº19889 du 9 juillet $2020^{2}$.

\section{LA NORME ET SON CONTEXTE}

L'article 392 dudit texte dispose que "Liberté de travail et droit de la direction de l'entreprise : " L'État garantit l'exercice pacifique du droit de grève, le droit des non-grévistes d'entrer et de travailler dans les établissements respectifs, et le droit de la direction des entreprises de pénétrer librement dans les locaux ». Cette disposition a suscité une certaine confusion au départ, car il est en effet inhabituel d'exiger que le droit de grève soit exercé " pacifiquement», établissant ainsi un traitement asymétrique par rapport aux autres droits en vigueur dans les relations collectives de travail (tels que l'exercice de la direction de l'entreprise, par exemple), ce qui semble indiquer un préjudice négatif évident à l'égard de l'exercice de la liberté syndicale.

Cette réglementation laconique de la grève contenue dans la loi n¹9889 doit être interprétée dans le contexte du système juridique de l'Uruguay, qui repose sur certaines traditions constituant une culture juridique très particulière et profondément enracinée, ainsi que du débat sur les modalités d'exercice de la grève, dont il faut rendre compte pour mieux comprendre le changement qui s'est produit.

Depuis des siècles, les relations collectives de travail en Uruguay jouissent d'un large éventail de libertés d'association. L'organisation des syndicats n'est soumise à aucune exigence administrative, à aucun enregistrement ni contrôle de l'État ; la négociation collective n'est limitée à aucune procédure ni à aucune règle fixe ${ }^{3}$, à l'exception de celle qui se déroulant dans les conseils sur les salaires, organes tripartites qui fixent les salaires par secteur d'activité ${ }^{4}$. Enfin, les grèves ne font l'objet d'aucune définition ou limitation légale, à l'exception de celles qui sont liées à la prestation de services essentiels.

Dans le cadre de la reconnaissance des droits collectifs, la grève inclut le recours à des ses modalités. L'occupation du lieu de travail comme mesure d'action collective est ainsi considérée comme un exercice du droit de grève dans le décret n 165 du 30 mai $2006^{5}$.

Cette remarquable extension des trois piliers de l'autonomie collective - organisation syndicale, négociation collective et grève - distingue très clairement le modèle uruguayen de la pratique dans les pays latino-américains limitant bien davantage les possibilités d'expression de la liberté d'association.

Cette ingérence minimale de l'État dans les relations collectives de travail a conduit à comparer I'Uruguay et l'Italie, ce qui a également eu pour conséquences que la doctrine juridique ait cherché à expliquer (et à soutenir) ce mode de fonctionnement du systèmé.

2 https://www.impo.com.uy/bases/leyes/19889-2020

3 Loi n¹8566 du 11 septembre 2009 : https://www.impo.com.uy/bases/leyes/18566-2009

4 Loi nº10449 du 12 novembre 1943 : https://www.impo.com.uy/bases/leyes/10449-1943

5 https://www.impo.com.uy/bases/decretos/165-2006

6 Pour un examen des raisons historiques, sociales et culturelles des caractéristiques du droit du travail en Uruguay : http://hugobarrettoghione.blogspot.com/2013/08/notas-sobre-elsurgimiento-del-derecho.html 
Cependant, certains traits du modèle uruguayen ont toujours suscité un " malaise » au sein des organisations d'employeurs, qui ont finalement déposé une plainte auprès du Comité de la liberté syndicale de l'Organisation internationale du travail (OIT), arguant :

- que la négociation collective tripartite sur les salaires portait atteinte à la liberté inscrite dans l'article 4 de la Convention internationale du travail $n^{0} 98^{7}$;

- que l'occupation des lieux de travail ne tenait pas compte de la liberté de travail des nongrévistes et du droit du propriétaire de l'entreprise d'entrer dans celle-ci pendant la durée de l'action, et qu'elle était donc considérée comme illégale.

Depuis 2010 et suite à cette plainte, la Commission d'experts pour l'application des Conventions et Recommandations (CEACR) surveille I'Uruguay, considérant que l'occupation des lieux de travail - en tant que mesure légitime - doit permettre l'accès aux locaux de l'entreprise aux non-grévistes et aux propriétaires de l'établissement ${ }^{8}$. Dans son rapport de 2019 (publié en 2020), la CEACR « appelle le gouvernement à (...) soumettre au Parlement un projet de loi réglementant l'occupation des entreprises de manière pleinement compatible avec la Convention ».

Il convient de noter que la CEACR, en considérant les occupations comme une forme de grève, demande au gouvernement uruguayen de les réglementer d'une manière qui soit « pleinement conforme à la Convention ». Elle admet ainsi que la Convention n`87 reconnaît la grève comme faisant partie de la liberté d'association, chose à laquelle l'Organisation internationale des employeurs s'est obstinément opposée et qu'elle nie depuis 2012, lorsqu'elle a fait valoir que la grève ne bénéficiait d'aucune reconnaissance internationale au sein de l'OIT.

Ainsi, le cas uruguayen revêt une grande importance dans la sphère de l'OIT puisque ce sont les employeurs eux-mêmes qui soumettent la question de la grève à la CEACR alors qu'en parallèle ils soutiennent, paradoxalement, que la grève ne jouit d'aucune reconnaissance internationale et, par conséquent, que la CEACR n'a rien à dire à ce sujet. Cette contradiction n'a pas d'issue logique.

\section{LES LIMITES DE L'INTERPRÉTATION}

Mais au-delà de cette contradiction évidente dans la position des employeurs au sein de I'OIT, force est de constater que depuis dix ans, l'Organisation demande à l'Uruguay de modifier sa réglementation sur les occupations afin d'harmoniser l'exercice du droit de grève sous forme d'occupation avec le droit de travail des non-grévistes et la liberté d'entreprise et de direction de l'employeur. Ces deux éléments, à savoir la tradition d'autonomie et les observations de l'OIT sur les grèves sous forme d'occupation, sont essentiels pour la lecture et l'interprétation de l'article 392 du règlement sur les grèves. Dès lors, l'article 392 de la loi n¹9889 ne peut pas être interprété comme une restriction de la grève puisque son

7 Voir à cet égard la dernière observation de la Commission d'experts pour l'application des conventions et recommandations de l'OIT: https://www.ilo.org/dyn/normlex/es/f?p=1000:13100:0 :NO:13100:P1310 0 COMMENT ID,P11110 COUNTRY ID,P11110 COUNTRY NAME,P11110 COMMENT YEAR:4023767,102876,Uruguay,2019

8 https://www.ilo.org/dyn/normlex/es/f?p=1000:13100:0::NO:13100:P13100 COMMENT ID,P11110 C ONTRY ID,P11110 COUNTRY NAME,P11110 COMMENT YEAR:3964045,102876,Uruguay,2018 
application même est destinée à encourager le progrès des droits sociaux et le bien-être de ceux qui travaillent pour les autres.

En résumé :

- Le caractère " pacifique » et compatible de la grève avec les autres droits relatifs à la liberté de travail et d'entreprise, tels que formulés à l'article 392 de la loi n¹9889, doit être interprété conformément à la Constitution qui à l'article 57 précise que toute réglementation doit veiller à garantir "l'efficacité » de la grève. La matrice indépendantiste du système juridique uruguayen va également dans ce sens, établissant dans son ensemble les limites d'interprétation du droit en vigueur ;

- Le libellé de l'article 392 de la loi d'urgence est pratiquement une copie conforme de certaines déclarations du Comité de la liberté syndicale (CLS), en particulier du n`940 dans sa dernière compilation", selon lequel «Le droit de grève doit s'exercer dans le respect de la liberté du travail des non-grévistes prévue par la législation nationale, ainsi que du droit de la direction de l'entreprise de pénétrer dans les locaux».

Cette reprise quasi littérale d'une déclaration du CLS marque une deuxième limite dans l'interprétation de l'article 392 : compte tenu de l'antécédent de la norme - les observations de la CEACR sur l'Uruguay- le nouveau dispositif légal est systématiquement expliqué dans le texte sur la conception de la grève de I'OIT, qui stipule au $n^{\circ} 784$ de la compilation précitée : "pour ce qui concerne les modalités du droit de grève refusées aux travailleurs (paralysies intempestives, grèves des bras croisés, grèves du zèle, grèves perlées, occupation de l'entreprise ou du lieu de travail), le Comité a considéré que ces limitations ne se justifieraient que si la grève perdait son caractère pacifique ».

\section{Conclusion}

L'approbation de l'article 392 ne devrait pas modifier de manière significative l'exercice du droit de grève si on l'interprète selon la directive constitutionnelle et conformément à la "doctrine " de la CEACR et aux Recommandations du CLS, qui sont les antécédents immédiats de la norme. Face à l'antinomie entre le droit de grève/liberté de travail et d'entreprise, l'article 392 empêche le recours exclusif à l'un ou l'autre; le texte devra donc être appliqué de manière à garantir l'efficacité de la grève, même s'il s'agit d'une occupation du lieu de travail. 